\title{
Impairment of pericardial leaflet structure from balloon-expanded valved stents
}

\author{
Wiebke de Buhr, DVM, PhD, ${ }^{\text {a }}$ Stefan Pfeifer, Dipl-Ing, ${ }^{\mathrm{b}}$ Julia Slotta-Huspenina, MD, $\mathrm{PhD},{ }^{\mathrm{c}}$ \\ Erich Wintermantel, MD, PhD, ${ }^{\mathrm{b}}$ Georg Lutter, $\mathrm{MD}, \mathrm{PhD},{ }^{\mathrm{d}}$ and Wolfgang A. Goetz, MD, $\mathrm{PhD}^{\mathrm{e}}$
}

Objective: Malpositioning is one of the major problems in transcatheter aortic valve implantation. To evaluate the influence of mechanical balloon inflation on aortic valve stent positioning, the expansion process and the impact on the valve leaflet's structure were investigated.

\begin{abstract}
Methods: Custom-made stents were laser cut from a 22-mm diameter stainless steel tube and mounted with a glutaraldehyde-treated bovine pericardial valve. The valved stents were crimped onto a standard balloon catheter and expanded by inflation of the balloon with 2 bar for 3 seconds. Expansion was studied using a high-speed camera, and the histology of the pericardial tissue was analyzed.
\end{abstract}

Results: The valved stents were fully expanded within 3 seconds. Balloon inflation was observed to be asymmetric starting proximally. At the beginning of expansion, the valved stents were pulled proximally. During further inflation, the stents slipped distally on the balloon and experienced a total displacement of $13.5 \mathrm{~mm}$. Macroscopic examination showed severe imprinting of the stent struts into the pericardial tissue. Histology revealed disrupted tissue layers and collagen fibers.

Conclusions: Analysis of valved stent expansion showed a displacement of the stent on the catheter during balloon inflation. Therefore, precise placement of the valved stent cannot be accomplished. Histologic analysis of the expanded pericardial tissue revealed disruption of collagen fibers. Disruption of pericardial tissue structures due to balloon expansion may result in early functional valve failure. (J Thorac Cardiovasc Surg 2012;143:1417-21)

Video clip is available online.

Two catheter-based aortic valve prostheses are currently available in Europe for transcatheter aortic valve implantation (TAVI): the Edwards SAPIEN Aortic Bioprosthesis (Edwards Lifesciences LLC, Irvine, Calif) and the Medtronic CoreValve System (Medtronic Inc, Minneapolis, Minn). The SAPIEN bioprosthesis consists of a stent made of a cobalt chromium alloy with glutaraldehydetreated bovine pericardial leaflets. The cobalt chromium

\footnotetext{
From the Central Laboratory Animal Facility, ${ }^{a}$ University Hospital Schleswig-Holstein, Campus Kiel, Kiel, Germany; Department of Medical Engineering, ${ }^{b}$ Technical University, Munich, Germany; Institute of Pathology, ${ }^{\mathrm{c}}$ University Hospital Rechts der Isar, Munich, Germany; Department of Cardiovascular Surgery, ${ }^{\mathrm{d}}$ University Hospital Schleswig-Holstein, Campus Kiel, Kiel, Germany; and Department of Cardiothoracic Surgery, ${ }^{\mathrm{e}}$ University Hospital, Regensburg, Germany.

Disclosures: Wolfgang Goetz is Managing Director of Transcatheter Technologies $\mathrm{GmbH}$, Regensburg, Germany. All other authors have nothing to disclose with regard to commercial support.

This study was performed at the Technical University of Munich's Department of Medical Engineering, Garching/Munich, Germany.

Received for publication Aug 21, 2011; revisions received Sept 28, 2011; accepted for publication Nov 7, 2011; available ahead of print Jan 13, 2012.

Address for reprints: Wolfgang A. Goetz, MD, PhD, University Hospital Regensburg, Department of Cardiothoracic Surgery, Franz Josef Strauß Allee 11, 93042 Regensburg (E-mail: wogoe@gmx.de)

$0022-5223 / \$ 36.00$

Copyright (C) 2012 by The American Association for Thoracic Surgery

doi:10.1016/j.jtcvs.2011.11.001
}

stent is crimped onto the balloon catheter just before implantation using a "crimper." The stent is expanded and placed by expanding the balloon inside of the valved stent. In contrast, the CoreValve System uses a self-expanding stent made of Nitinol memory metal with glutaraldehydetreated porcine pericardial leaflets. The Nitinol stent is crimped to a small diameter in ice water and pushed into the catheter sleeve just before implantation. When retracting the sleeve, the Nitinol stent expands and self-anchors in the aortic root. ${ }^{1}$ We investigated the behavior of a custom-made, steel, balloon-expandable valved stent during the process of balloon expansion.

For more than 20 years, it has been well known that mechanical stress and disruption of collagen fiber structure in prosthetic valves leaflets initiate calcification of the leaflet tissue and cause early valve deterioration. ${ }^{2}$ Functional and mechanical stress factors are assumed to contribute to calcification. $^{3-5}$

In 1990, Thubrikar $^{6}$ summarized the effects of stresses on biological tissues used as heart valve prostheses. Stress in pericardial tissue causes delamination of the tissue layer and disruption of collage fiber structure, both of which result in leaflet calcification and early valve deterioration.

The pericardial tissue used in catheter-based valve prostheses undergoes compression when the stent is crimped onto the deflated balloon and then again undergoes compression and high stress when the stent is expanded with 


\section{Abbreviation and Acronym}

TAVI $=$ transcatheter aortic valve implantation

the high-pressure balloon. We investigated the possibility of impairment of the fragile pericardial tissue during this process.

\section{MATERIALS AND METHODS \\ Stent}

Custom-made stents with 2 different closed-cell designs were laser cut from a $22-\mathrm{mm}$ diameter stainless-steel tube bearing a wall thickness of $0.5 \mathrm{~mm}$ and a height of $16 \mathrm{~mm}$. One stent design included only a diamond shape (Figure 1,A), and one stent design included vertical struts (Figure $1, B$ ). To ensure an even surface, the stents were polished after the laser cutting process.

\section{Pericardium}

Calf pericardium was harvested at a public abattoir, where it was stored in cold water $\left(4^{\circ} \mathrm{C}\right)$ and immediately transferred to the laboratory. Careful cleaning was performed to not disrupt the pericardial structure with instruments. The pericardium was then carved to produce a tension-free, flat, 0.7- to 0.8 -mm-thick specimen that was placed in a flat basin at room temperature and treated with $0.65 \%$ buffered glutaraldehyde for 7 days. The pericardium was trimmed into rectangular strips according to the height and circumferential distance of the stent used (stent 1 was $70 \times 20 \mathrm{~mm}$ ). Ten pericardial strips were then mounted in the stents: 5 strips in each stent design (Figure 1, $A$ and $B$ ).

\section{Balloon}

The valved stent was crimped onto a standard balloon catheter (25.0 mm, NuCLEUS; NuMED, Inc, Hopkinton, NY). For expansion, the balloon was inflated with a 50-mL, saline-filled syringe using the standard procedure for in vivo TAVI. The balloon was dilated with a maximum pressure of $\mathrm{p}_{\max }=2$ bar within 1 second, and the pressure was held for 3 seconds (Figure 2). The entire expansion procedure was digitally recorded with a high-speed camera (HCC 1000; VDS, Osnabrück, Germany) using ImageJ software. ${ }^{7}$ In our captured images, the software's pixel length of

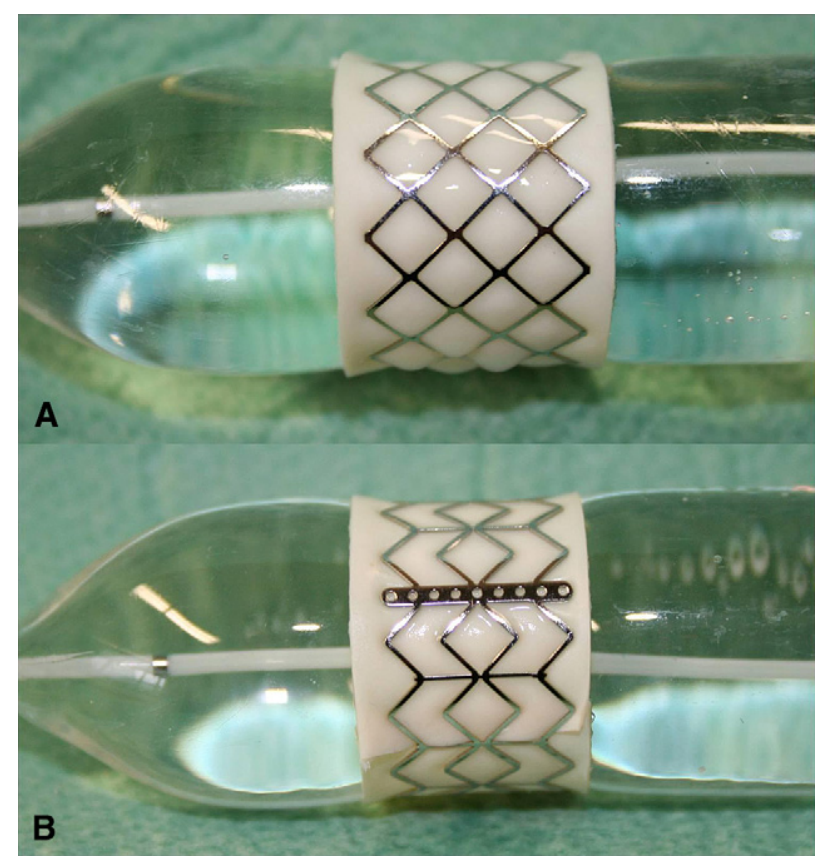

FIGURE 2. Expanded stents (A, stent 1; B, stent 2) during balloon inflation with 2 bar for 3 seconds.

$14.5 \mathrm{~mm}$ corresponding to 295 pixels was calibrated on the basis of the lengths of the stent strut and the stitch holes.

\section{Histology}

Immediately after the expansion procedure, the pericardium was uncoiled from the stents and macroscopically analyzed. For histologic examination, the specimens were preserved in $10 \%$ phosphate-buffered formalin for 2 days, processed and embedded in a conventional tissue processor (Shandon Pathcentre; Thermo Scientific, Dreieich, Germany), where they underwent dehydration through serial bath treatments containing xylol and ethanol, and finally embedded in paraffin. The formalin-fixed, paraffin-embedded tissue was cut into $4-\mu \mathrm{m}$-thick slices using a rotary microtome (Leica RM2135; Leica Microsystems GmbH, Wetzlar, Germany) in longitudinal sections through the pericardial conduit, longitudinal

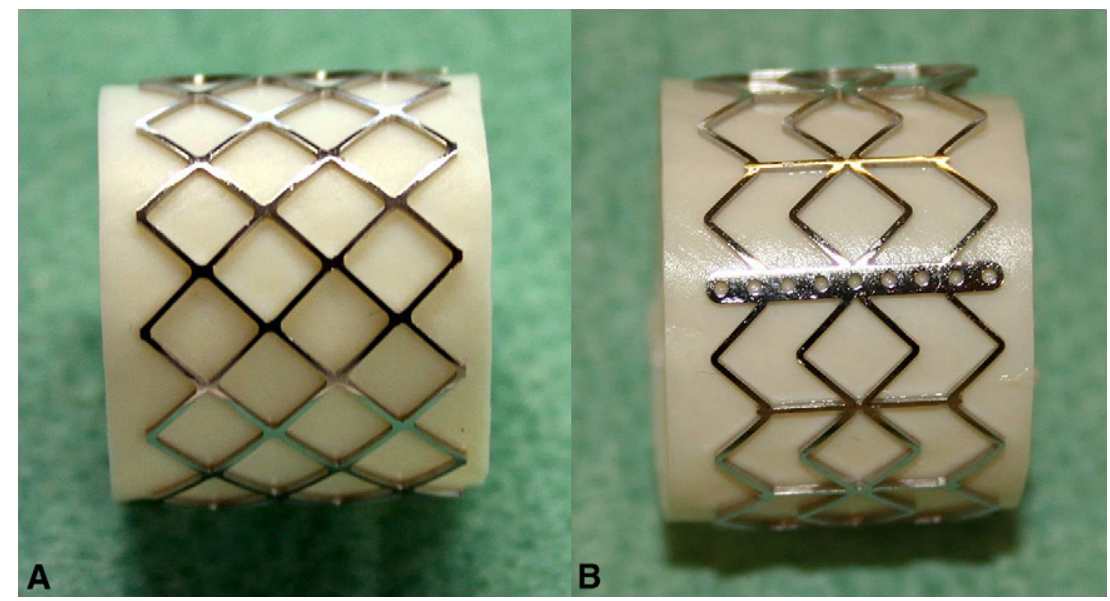

FIGURE 1. A, Stent 1: stainless-steel stent with diamond-shaped cells and bovine pericardium attached. B, Stent 2: stainless-steel stent with 3 commissural struts and bovine pericardium attached. 


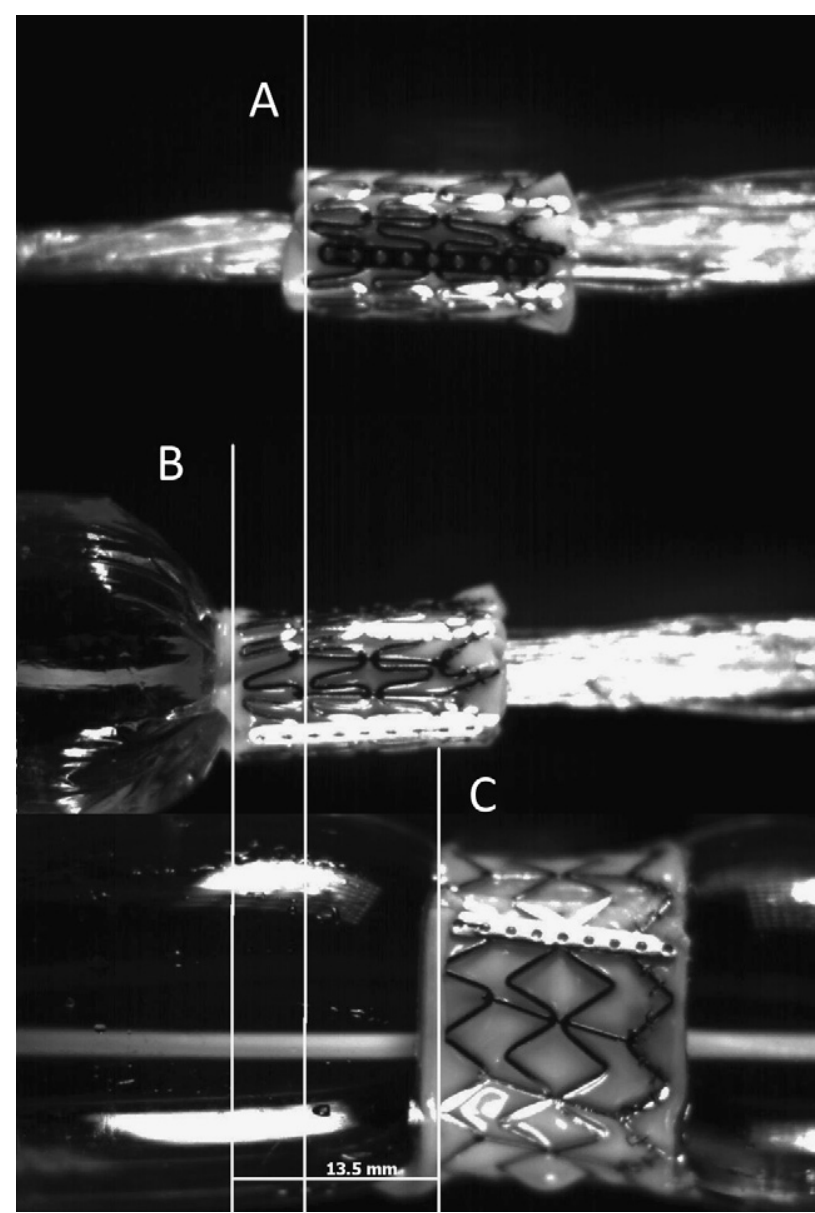

FIGURE 3. The image sequence shows the sliding movement of valved stent number 2 during its expansion: A, The starting position of the stent is line A; B, During inflation, the stent migrated $4.5 \mathrm{~mm}$ in the proximal direction to line B; C, At the end of balloon dilation, the direction switched, and the valved stent slid to the distal position (line $\mathrm{C}$ ) with a maximum displacement of $13.5 \mathrm{~mm}$. A slow-motion video of the camera sequence that ends on this frame can be seen in Video 1.

sections through the middle of each cusp, and a transverse section through each commissure. Representative sections were stained with Elastica-van Gieson (Resorcin-Fuchsin) for collagen morphology.

\section{RESULTS}

\section{Expansion}

To present the main focus of attention during expansion, a selection of high-speed images is shown (Figure 3; Video 1). The expansion procedure was divided into 2 periods: (1) At the beginning of expansion, only the proximal part of the balloon inflated, because the crimped stent prevented the injected fluid from flowing freely to the distal part of the balloon. This asymmetric balloon expansion proximally displaced the valved stent (Figure 3, distance A to B). (2) After the proximal part of the balloon was fully inflated, the expanding balloon pushed the valved stent distally, and the valved stent slid to the distal end of the

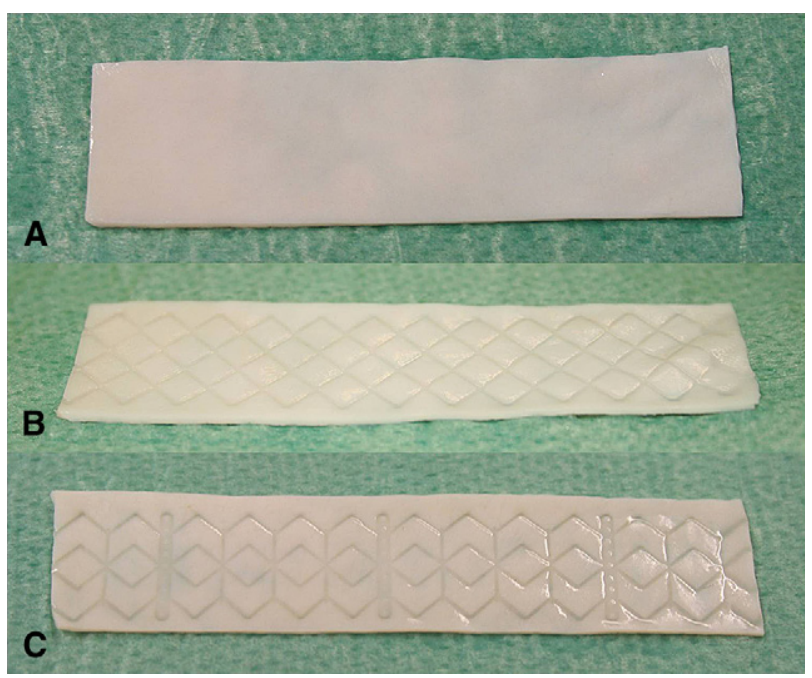

FIGURE 4. A, Native pericardium trimmed into a rectangular strip before being sutured into the stent. B, Unrolled pericardium after stent 1 expansion with a pressure of 2 bar for 3 seconds. C, Unrolled pericardium after stent 2 expansion with a pressure of 2 bar for 3 seconds. Note the imprinted shape of both stents onto the pericardium, like a "skid mark."

catheter (Figure 3, distance B to C). Both valved stents were expanded within 1 second with a pressure of 2 bar, and the pressure was held for 3 seconds. For the displacement, we measured a maximum value of $4.5 \pm 0.1 \mathrm{~mm}$ between line A and B and $13.5 \pm 0.1 \mathrm{~mm}$ between $\mathrm{B}$ and $\mathrm{C}$.

\section{Pericardium}

During stent expansion by balloon inflation with a pressure of 2 bar, the pericardium was pressed against the stent struts for 3 seconds. Macroscopic analysis showed that compression of the pericardium against the stent structure resulted in an imprinted "skid mark" (Figure 4).

\section{Histology}

Elastica-van Gieson staining was used to visualize the general collagen morphology of the pericardium. In all 10 histologic samples $(\mathrm{n}=5$ of Figure $1, A$ and $\mathrm{n}=5$ of Figure $1, B)$, broken collagen fibers were observed at 2 levels: The tissue surface was disrupted and the superficial collagen fibers were broken at the corner of the stent-strut indent, and collagen fibers were disrupted at the edge of the indent along an oblique line into deeper situated layers (Figure 5). No differences were observed between the 2 groups (Figure 1, $A$ and $B$ ).

\section{DISCUSSION}

Correct valve positioning is crucial for successful TAVI, but as Kapadia and Tuzcu ${ }^{8}$ stated in 2009, major complications of TAVI include device malpositioning. Optimal angiographic projection of the aortic annulus is essential for accurate TAVI positioning. ${ }^{9}$ Nevertheless, the accuracy of 


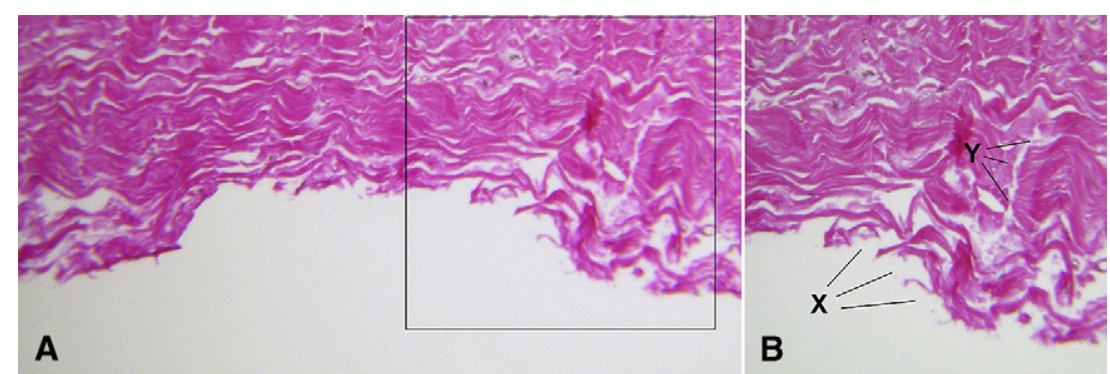

FIGURE 5. A, Histology of the pericardium indented by the stent's struts. B, Histology of the boxed area from A ( $X=$ disrupted surface and broken superficial collagen fibers; $Y=$ broken collagen fibers in the deeper layers of the pericardial leaflet).

positioning depends mostly on the skills of the physician. In 2009, Jilaihawi and coworkers ${ }^{10}$ described optimal positioning in $50 \%$ of their cases, whereas Zajarias and Cribier $^{11}$ reported tolerable placement in $92.8 \%$ of their cases. However, remaining paravalvular leakage in more than $50 \%$ of the implantations due to suboptimal positioning has also been reported. ${ }^{12}$ Placement of a second valve (valve-in-valve) is required in up to $3 \%$ of cases to correct a prior valve malposition. ${ }^{13}$

Our results show that the skill of the implantation team is not the only relevant factor in correct valved stent positioning. Another factor (revealed by high-speed camera) that should be taken into consideration is the displacement of the valve caused by asymmetric balloon expansion. The asymmetric nature of the expansion of the stented valve on the balloon leads to displacement of the valved stent on the catheter. This acute migration of the valved stent impedes precise positioning of the valve.

The TAVI valve prosthesis is crimped onto the balloon right before it is implanted. ${ }^{14}$ For valve deployment, the delivery balloon is inflated to expand the valved stent, thereby compressing the native aortic valve against the stent structure.

Histologic examination of the expanded pericardial tissue revealed strut imprints on the valve tissue with disrupted leaflet surface structures and broken collagen fibers. The disruption of the leaflet surface structures is expected to lead to a higher risk of early valve thrombosis. ${ }^{15,16}$ In pericardial bioprostheses, calcification is found along the shear planes where the structural integrity has been disrupted by individual layers of collagen sliding over each other and where mechanical stresses damaged the structural integrity of the leaflet tissue. ${ }^{2}$

The damage of tissue structures caused by balloon expansion that was obvious in our experiment is expected to cause early leaflet calcification, resulting in early functional valve failure.

Zegdi and colleagues ${ }^{17}$ recently published a clinical study that revealed traumatic injury to leaflets after percutaneous valve implantation. Our study confirms these findings and demonstrates a possible cause for the observed pericardial leaflet injury.

\section{Study Limitations}

The custom-made stents in this experiment are not fully comparable to the commercially available stents used for valve implantation. The stents used in this experiment were made of stainless steel, whereas the commercially available balloon expandable stents are made of cobalt chromium alloy (Edwards SAPIEN Aortic Bioprosthesis) and Nitinol (Medtronic CoreValve). The material properties surely differ and were not investigated in this study. Therefore, our results cannot be directly applied to the commercially available devices. In addition, the valve leaflet design in our custom-made stents was a simple strip of pericardium and differs from the valve design in the commercially available prostheses.

We did not investigate or differentiate whether the crimping alone or the expansion process alone caused injury to the pericardial leaflets.

The in vitro experimental model we used differs from a real clinical scenario. When the valve frame is balloon expanded in a stenotic and calcified aortic root, the stent has a large contact area with the native valve leaflets, and this may reduce the described effect of stent displacement caused by the asymmetric balloon dilation.

Finally, this study was not intended to investigate the long-term effects of the observed leaflet injury.

\section{CONCLUSIONS}

Analysis of valved stent expansion showed asymmetric balloon inflation, which displaces the stent during expansion. This movement prevents precise placement of balloon-expandable valved stents. Histologic analysis of expanded pericardial tissue revealed strut imprints on the valve tissue, disruption to the surface of the tissue, and disrupted collagen fibers. Disruption of tissue structures due to balloon expansion may result in early functional valve deterioration. Further investigation regarding the clinical relevance of the observed tissue injury is essentially mandatory. 


\section{References}

1. Leon M, Kodali S, Williams M, Oz M, Smith C, Stewart A, et al. Transcatheter aortic valve replacement in patients with critical aortic stenosis: rationale, device descriptions, early clinical experiences, and perspectives. Semin Thorac Cardiovasc Surg. 2006;18:165-74.

2. Thubrikar MJ, Deck JD, Aouad J, Nolan SP. Role of mechanical stress in calcification of aortic bioprosthetic valves. J Thorac Cardiovasc Surg. 1983;86: 115-25.

3. Cunanan CM, Cabiling C, Dinh T, Shen S, Tran-Hata P, Rutlege J, et al. Tissue characterization and calcification potential. Ann Thorac Surg. 2001;71: 417-21.

4. Schoen FJ, Levy RJ. Calcification of tissue heart valve substitutes: progress toward understanding and prevention. Ann Thorac Surg. 2005;79:1072-80.

5. Reul H, Potthast K. Durability/wear testing of heart valve substitutes. J Heart Valve Dis. 1998;7:151-7.

6. Thubrikar MJ. Replacement cardiac valves. III. Bioprothesis. In: The aortic valve. Boca Raton: CRC Press, Inc; 1990.

7. Rasband WS. ImageJ. Bethesda, MD: US National Institutes of Health; 19972011. Available at: http://imagej.nih.gov/ij/. Accessed November 11, 2011.

8. Kapadia SR, Tuzcu EM. Transcatheter aortic valve implantation. Curr Treat Options Cardiovasc Med. 2009;11:467-75.

9. Cribier A, Litzler P, Eltchaninoff H, Godin M, Tron C, Bauer F, et al. Technique of transcatheter aortic valve implantation with the Edwards-Sapien ${ }^{\circledR}$ heart valve using the transfemoral approach. Herz. 2009;34:347-56.
10. Jilaihawi H, Chin D, Spyt T, Jeilan M, Vasa-Nicoteray, Bence J, et al. Prosthesispatient mismatch after transcatheter aortic valve implantation with the Medtronic-Corevalve bioprosthesis. Eur Heart J. 2010;31:857-64.

11. Zajarias A, Cribier AG. Outcomes of percutaneous AVR. J Am Coll Cardiol. 2009;53:1829-36.

12. Piazza N, Grube E, Gerckens U, Den Heijer P, Linke A, Luha O, et al. Procedural and 30-days outcomes following transcatheter aortic valve implantation using the third generation (18Fr) CoreValve ReValving System: results from the multicentre, expanded evaluation registry 1-year following CE mark approval. EuroIntervention. 2008;242-9.

13. Maisano F, Michev I, Denti P, Alfieri O, Colombo A. Transfemoral transcatheter aortic valve implantation using the balloon expandable SAPIEN transcatheter heart valve device. Multimedia Manual Cardiothorac Surg [serial online]. June 26, 2008;3087-95. Available at: http://mmcts.ctsnetjournals.org/cgi/content/ full/2008/0626/mmcts.2007.003087. doi:10.1510/mmcts.2007.003087.

14. Webb JG, Pasupati S, Humphries K, Thompson C, Altwegg L, Moss R, et al. Percutaneous transarterial aortic valve replacement in selected high-risk patients with aortic stenosis. Circulation. 2007;116:755-63.

15. Schoen F, Hirsch D, Bianco R, Levy R. Onset and Progression of calcification in porcine aortic bioprosthetic valves implanted as orthotopic mitral valve replacements in juvenile sheep. J Thorac Cardiovasc Surg. 1994;108:880-7.

16. Glasmacher B, Reul H, Schneppershoff F, Schreck S, Rau G. In vitro calcification of pericardial bioprostheses. J Heart Valve Dis. 1998;7:415-8.

17. Zegdi R, Bruneval P, Blanchard D, Fabiani JN. Evidence of leaflet injury during percutaneous aortic valve deployment. Eur J Cardiothorac Surg. 2011;40:257-9. 